\title{
La aplicación de Google Earth para la Educación Patrimonial en Ciencias Sociales
}

Ma Pilar Molina Torres. Universidad de Córdoba

Recepción: 10 de junio de 2017 | Aceptado: 17 de julio de 2017

Correspondencia: Ma Pilar Molina Torres | Correo-e: pilar.molina@uco.es

\section{iD 0000-0001-7157-0324}

Citar: Molina Torres, M.P. (2017). La aplicación de Google Earth para la Educación Patrimonial en Ciencias Sociales. ReiDoCrea, 6, 221-228.

Resumen: Pese al interés que suscita el conocimiento del Patrimonio Histórico, su presencia en el currículo es imperceptible desde el punto de vista documental y material. Mediante la puesta en práctica de un recorrido didáctico en Google Earth diseñado por el alumnado de tercer curso del Grado de Educación Primaria de la Universidad de Córdoba y desarrollado en la asignatura de Didáctica de las Ciencias Sociales, pudimos conocer el entorno del río Guadalquivir y el patrimonio natural y cultural a su paso por el casco urbano de Córdoba. La metodología utilizada fue esencialmente investigativa, a partir de la cual los alumnos pudieron obtener información del itinerario escogido y obtener sus propias hipótesis y resultados de la relación existente entre patrimonio y paisaje historizado. Asimismo, la propuesta tuvo como resultado la concienciación y el fomento de la conservación, no solo de bienes culturales sino también de los naturales.

Palabras clave: Aprendizaje activo | Paisaje cultural

The application of Google Earth for Heritage Education in Social Sciences

Abstract: In spite of the interest aroused by the knowledge of Historical Heritage, its presence in the curriculum is imperceptible from the documentary and material point of view. Through the implementation of an educational tour in Google Earth designed by third year students in the Primary Education Degree of the University of Cordoba and developed in the Didactics of Social Sciences course, we were able to get to know the environment of the Guadalquivir river and the natural and cultural heritage as it passes through the urban area of Córdoba. The methodology used was essentially investigative, by which the students were able to obtain information about the itinerary chosen and formulate their own hypotheses and results of the relationship between heritage and historicized landscape. The proposal also resulted in awareness and promotion of conservation, of natural as well as cultural heritage.

Keywords: Active Learning | Cultural Landscape

* La investigación que se presenta en las siguientes líneas es resultado de la implementación del Proyecto de Coordinación para la Mejora de la Calidad Docente titulado: "Proyecto de coordinación para la innovación en la Facultad de Ciencias de la Educación" de la Universidad de Córdoba, durante el curso 2015/2016.

\section{Introducción}

La presente investigación parte de la escasez de metodologías que utilizan las TIC para estudiar el patrimonio histórico y natural, dado que en la mayor parte de los centros educativos la educación patrimonial no se trata de manera específica como contenido. De hecho, en opinión de Viñao, el patrimonio es algo valioso que se hereda o que nosotros mismos construimos a lo largo de nuestra vida (2010, p. 17 ss.). Por tanto, es algo que se considera propio en el sentido de que forma parte de lo que nos pertenece, de lo que creemos nuestro. Con frecuencia, los aspectos patrimoniales en la etapa de Educación Primaria aparecen en un plano secundario pasando desapercibidos, y de manera sucinta en los contenidos curriculares. Sin embargo, su 
tratamiento está cobrando actualmente gran relevancia tanto a nivel nacional como autonómico.

La enseñanza del espacio patrimonial y natural se encuentra marcada por una serie de cambios y transformaciones que genera en la práctica docente una línea de investigación poco atendida por la historiografía. Por lo tanto, el objetivo de este trabajo se centra en fomentar la concienciación hacia el patrimonio histórico y facilitar su acercamiento desde nuestras propias experiencias. De hecho, la educación patrimonial se presenta como un elemento educativo que puede y debe ser aprendido por los futuros docentes, y en nuestro caso, concretamente por los maestros del Grado de Educación Primaria.

La finalidad de llevarlo a la escuela proporciona ciudadanos y docentes capaces de producir recursos educativos que involucren no solamente a los estudiantes, sino a la sociedad a través de valores como la educación ambiental (Martín, 1995; Antón, 2002; Beltz, 2002 y Novo, 2003). En este contexto, la propuesta se desarrolló en tres fases: por un lado, se realizaron diferentes recursos para reorganizar los conocimientos previos que tenía el alumnado universitario acerca de la educación patrimonial. En una segunda fase, el trabajo se centró en el diseño del itinerario didáctico y en consecuencia de la investigación relacionada con los recursos naturales y patrimoniales del entorno del río Guadalquivir a su paso por Córdoba. Finalmente, se expusieron los resultados conseguidos con la ruta diseñada a los compañeros de otros cursos en la asignatura de Didáctica de las Ciencias Sociales.

Desde esta perspectiva, según nos dice Ávila: "derivados de la enseñanza por descubrimiento dirigido, están los itinerarios didácticos basados en la investigación que caminan hacia un proceso de innovación en la didáctica del Patrimonio históricoartístico" (2007, p. 37). Por este motivo, hay que señalar que para favorecer las salidas es necesario contextualizar al alumnado con actividades que le sean cercanas y potencien los instrumentos didácticos que se utilizarán para planificar las rutas y señalar las zonas de interés. De ahí que el uso del Google Earth permita el diseño de itinerarios vinculados a una metodología activa y al interés de los participantes por investigar el territorio seleccionado (Córcoles, 2010).

De hecho, en la actualidad el tratamiento de la Educación Patrimonial con el alumnado de Primaria no va más allá de los contenidos histórico-artísticos recogidos en los manuales escolares de Ciencias Sociales. En muchos casos, las actividades que se desarrollan en la práctica se llevan a cabo sin tener conocimiento del entorno cultural de un ámbito urbano o rural. Partiendo de esta premisa, nuestro principal objetivo ha sido la utilización de una aplicación TIC para que los docentes puedan poner en práctica con sus alumnos rutas didácticas y conocer su entorno patrimonial más próximo. Asimismo, su contacto con el patrimonio histórico y natural del entorno del río Guadalquivir permitió concienciar a los estudiantes de su responsabilidad para conservarlo y valorarlo. En definitiva, hemos pretendido formar a los futuros docentes de Educación Primaria en la adquisición de competencias para interpretar los paisajes históricos y su combinación con herramientas digitales.

\section{Método}

\section{Participantes}

Para este estudio realizamos una salida con un grupo de cincuenta y tres estudiantes de tercer curso del Grado de Educación Primaria de la Universidad de Córdoba, quienes habían diseñado previamente ese itinerario a través de una aplicación TIC. De 
este modo, pudieron conocer el entorno patrimonial que les rodea y, en consecuencia, adquirir la posibilidad de enseñarlo en las aulas de Primaria, lo que sería de gran ayuda para los docentes implicados en la educación patrimonial. Esta experiencia didáctica que se abordó desde una metodología constructivista necesitó para su implementación de la recopilación de información del entorno natural-cultural del río Guadalquivir, delimitación de la ruta con Google Earth, utilización y consulta de fotos antiguas y actuales, etc. Todo ello con la finalidad de elaborar diversos materiales didácticos que les sirvieran para conocer el patrimonio de nuestra ciudad y asimismo poder utilizarlos como futuros docentes. Los resultados demostraron las dificultades que los estudiantes tienen para interpretar los elementos patrimoniales que les rodean y analizar el entorno cultural elegido.

\section{Instrumentos}

Este proyecto nace con la idea de compartir diferentes rutas didácticas entre los docentes de la Comunidad Andaluza. De esta forma, conseguiremos proporcionar una herramienta de fácil manejo para el docente que esté interesado en realizar actividades en un entorno concreto con un grupo de alumnos de Educación Primaria (Figura 1).

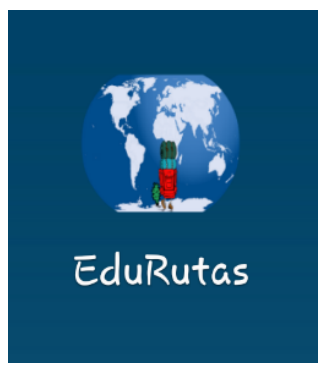

Figura 1. Proyecto EduRutas.

La aplicación cuenta con una interfaz en la que aparecen tres botones: "FECHA", "HORA" y un tercero, "MIS RUTAS", que al pulsarlo nos permite acceder al siguiente menú en el que se muestran las diferentes provincias de la Comunidad Autónoma de Andalucía. Es importante esta clasificación en provincias porque permite localizar la ruta que se busca en menor tiempo y con más facilidad de acceso. En este caso, aparece este menú como prototipo, pero igualmente podría adaptarse de forma que dentro de cada provincia aparecieran otros submenús relacionados con comarcas e incluso si el uso se hiciera más frecuente en una localidad en concreto, estructurarlo de forma que se organizaran los parajes de dicha localidad. Por ejemplo, en Córdoba, estructurar submenús en torno a Sierra, Subbética, Campiña, o también a nivel local organizar los diferentes recorridos por cada una de sus fuentes, o parajes agrícolas, etc. A modo de ejemplo, solo lo hemos reflejado con rutas Córdoba (Figuras 2 y 3 ).

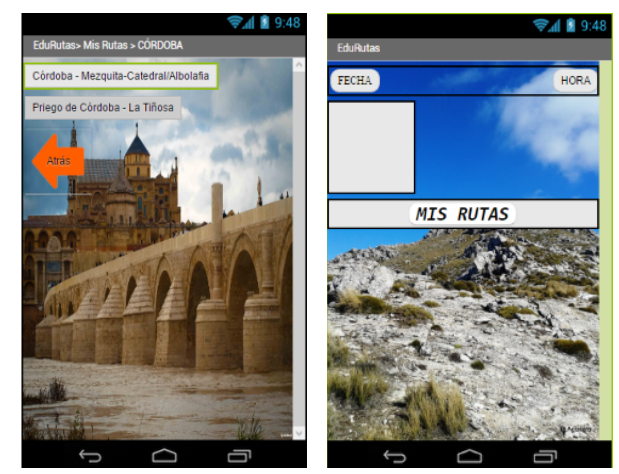

Figuras 2 y 3 . Captura de pantalla de la aplicación MIS RUTAS. 
La aplicación está formada por cada una de las diferentes rutas que los docentes quieran incluir y compartir con el resto de la comunidad educativa. Consiste en plasmar itinerarios didácticos sobre lugares completándolos con actividades para realizar antes, durante y después de la salida. El hecho de crear la ruta en Google Earth hace que tengamos acceso a una serie de datos básicos y muy valiosos para practicar la ruta con nuestros alumnos. Así, de esta manera, podemos ver la longitud, perfil del relieve, tiempo de duración, tiempo atmosférico en su desarrollo, ver las zonas del mapa en las diferentes estaciones o incluso su evolución en periodos de tiempo de años o décadas, analizando una posible evolución histórica del terreno y por consiguiente disponer la visita en función de la edad de los alumnos en cuestión.

Además, una de las finalidades de esta aplicación es que nuestro trabajo y experiencia de campo puedan ser válidos para otros docentes, ya que nuestros conocimientos e investigaciones hacen que la aplicación se complete y al estar compartida en "EduRutas" facilitamos encontrar rutas de lugares desconocidos para otros docentes, a la vez que se ofrecen diferentes perspectivas que pueden pasar desapercibidas para quien no las conoce. En cuanto a las actividades, es conveniente desarrollarlas antes de la visita en la que se presenta a los alumnos cómo afrontar la excursión o el recorrido en clase, dar algunos datos de interés, e incluso preparar un cuaderno de viaje y cómo completarlo adecuadamente. Por tanto, planificamos cada una de las partes de la salida y asimismo concretamos las diferentes maneras de afrontarlas.

Durante el itinerario se desarrollarán las paradas que aparecen en la ruta en la que se incluirán datos para compartir con el alumnado, ya sean históricos, geográficos, turísticos (Figura 4). Éstos se recogerán en una ficha con diferentes preguntas o en el propio diario de viaje, que se completarán con fotos de los alumnos o dibujos de cada uno de los diferentes lugares visitados. Finalmente, tras la visita, se puede llevar a cabo una exposición individual o por grupos de cada una de las características principales que han encontrado en el viaje, si les ha gustado, si lo recomendarían y similares. También, si incluirían algo más a la salida didáctica, investigando en su casa con anterioridad a la exposición oral.

Desde el punto de vista del docente, se podría introducir una valoración de las rutas finalizadas o algún comentario en la aplicación o foros para compartir las experiencias. Se podrían completar o añadir rutas realizadas por docentes, ya que el fin de la aplicación no solo es obtener esos itinerarios, también se pueden incluir recorridos nuevos de cada una de las diferentes provincias, ciudades o pueblos de la Comunidad Andaluza, enriqueciendo su utilidad con diversos datos proporcionados por los interesados y expertos en la materia puesta en práctica.

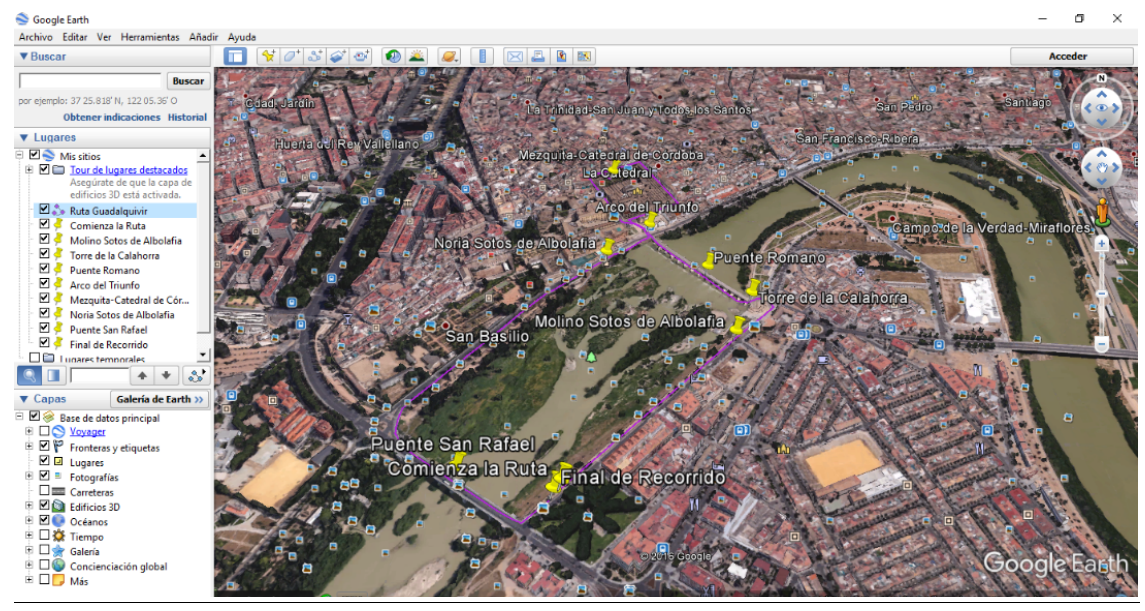

Figura 4. Diseño del itinerario realizado por los alumnos. 
Por otra parte, para evaluar la adquisición de dichas competencias educativas y preguntarnos si la puesta en práctica del proyecto estaba acorde con los intereses y necesidades de los alumnos del Grado de Educación Primaria, se les entregó a los cincuenta y tres participantes un cuestionario para autoevaluarse y detectar si el trabajo realizado había sido valorado como un recurso útil para su futura práctica docente (Tabla I).

\begin{tabular}{|c|c|c|c|}
\hline Tabla I. Cuestionario individual. & & & \\
\hline Aspectos a valorar & SI & NO & $\mathrm{NS} / \mathrm{NC}$ \\
\hline $\begin{array}{l}\text { 1. ¿Te ha parecido adecuada la aplicación de Google Earth } \\
\text { para diseñar la ruta didáctica? }\end{array}$ & & & \\
\hline 2. ¿Conoces más sobre el patrimonio de tu ciudad? & & & \\
\hline $\begin{array}{l}\text { 3. ¿Pondrías en práctica estos itinerarios en tu futura práctica } \\
\text { como docente? }\end{array}$ & & & \\
\hline 4. ¿Recomendarías a otros docentes que la utilicen? & & & \\
\hline $\begin{array}{l}\text { 5. ¿Es útil el proyecto EduRutas para la comunidad } \\
\text { educativa? }\end{array}$ & & & \\
\hline $\begin{array}{l}\text { 6. ¿Dicho proyecto da a conocer el patrimonio cultural y } \\
\text { natural de una zona determinada? }\end{array}$ & & & \\
\hline 7. ¿Te parece un recurso motivador para los alumnos? & & & \\
\hline
\end{tabular}

\section{Procedimiento}

El estudio que tuvo dos fases: combinó una primera parte con el aprendizaje y posterior utilización en el aula de la aplicación TIC, y una segunda de puesta en práctica de esa primera fase, pero de trabajo en campo. Fueron necesarias varias salidas al lugar que queríamos contextualizar en las rutas. Para ello, se conformaron grupos de 5 ó 6 alumnos para analizar los recursos naturales y los elementos patrimoniales que complementan el conjunto de bienes culturales y medioambientales. Dispusimos de la hora y media semanal de prácticas para la asignatura de Didáctica de las Ciencias Sociales, dividiendo a los cincuenta y tres alumnos en dos grupos de 26/27, y a su vez en pequeños subgrupos de 5 ó 6 estudiantes. Tras su investigación en las zonas indicadas en Google Earth, elaboraron un dossier con toda la información más relevante acerca del paisaje, explotación de recursos de la zona elegida, transformación del entramado urbano y acondicionamiento de los monumentos al urbanismo actual. Esta investigación grupal supuso un trabajo más eficaz, autónomo e interdisciplinar en el momento que tuvieron que volcar los diferentes resultados investigativos en la aplicación didáctica y comprobar que habían utilizado una herramienta muy valiosa para su labor docente y la actual de muchos compañeros.

A continuación, se estableció un sistema de autoevaluación individual que se realizó en una sesión de prácticas en la Universidad. Contamos con hora y media para validar su experiencia y la información aportada al proyecto. Las ideas previas se sondearon a mano alzada para conocer el interés personal/grupal de los trabajos en equipo. Cuestiones como: ¿consideras el trabajo en equipo esencial en estos proyectos?, o ¿has tenido dificultades para trabajar con las TIC?, fueron cuestiones planteadas en clase que tuvieron una respuesta afirmativa a nivel de grupo y que valoraron como positiva para su formación docente. Con posterioridad, a este sondeo oral se utilizó el aula donde se imparte la docencia y se explicó cómo debían resolver los diferentes ítems. Tomada la muestra del estudio se efectuó un recuento de las respuestas para analizar en su conjunto los datos e interpretar las conclusiones de dicha prueba. 


\section{El análisis de los datos}

Este proyecto didáctico nos ofrece un amplio abanico de posibilidades para conseguir los objetivos planteamos al principio. A partir de la muestra tomada planteamos una investigación cualitativa que evaluó las experiencias personales de los estudiantes acerca del espacio natural y cultural que se representó en la aplicación TIC, su delimitación con la utilización de Google Earth, y la puesta en práctica del itinerario con la ruta elegida. De esta manera, las diferentes cuestiones abordadas en el cuestionario aportaron una posición esencialmente subjetiva de los maestros a la hora de configurar estos recorridos didácticos y su posterior salida con los alumnos de Educación Primaria. También a través de EduRutas tuvieron la posibilidad de comparar y planificar los recursos históricos y naturales de su ciudad con otros muchos que pudieron conocer a través de la aplicación con Google Earth. Del mismo modo, mediante el análisis de las respuestas se evidencia que el diseño de estas rutas didácticas es una actividad básica en la formación del profesorado y que desde el punto de vista interdisciplinar permiten poner en práctica las competencias educativas recogidas en la legislación actual.

\section{Resultados}

Del recuento de las respuestas pudimos extraer varias conclusiones y analizar la información individual y cualitativa de la implementación del proyecto. De las siete cuestiones planteadas a los cincuenta y tres alumnos fue significativo como la mayoría de respuestas eran afirmativas, lo que mostraba a un alumnado convencido de que la herramienta utilizada para realizar itinerarios didácticos era útil y necesaria en su práctica como futuros docentes. Sin embargo, y aunque se utilizó en el entorno natural y cultural del río Guadalquivir, la cuestión 2 muestra cierta indecisión ante los conocimientos volcados en EduRutas y el aprendizaje personal del alumno para conocer más sobre el patrimonio de la ciudad. Asimismo, destaca la respuesta 7 relacionada con el recurso aplicado y su carácter motivador. De hecho, aproximadamente un $32 \%$ del grupo piensa que no sabe o no contesta si realmente sería un recurso motivador para el alumnado de Primaria. Por tanto, este significativo resultado podría indicarnos la reflexión de nuestros alumnos del Grado de Educación Primaria y en consecuencia su falta de experiencia en este tipo de proyectos.

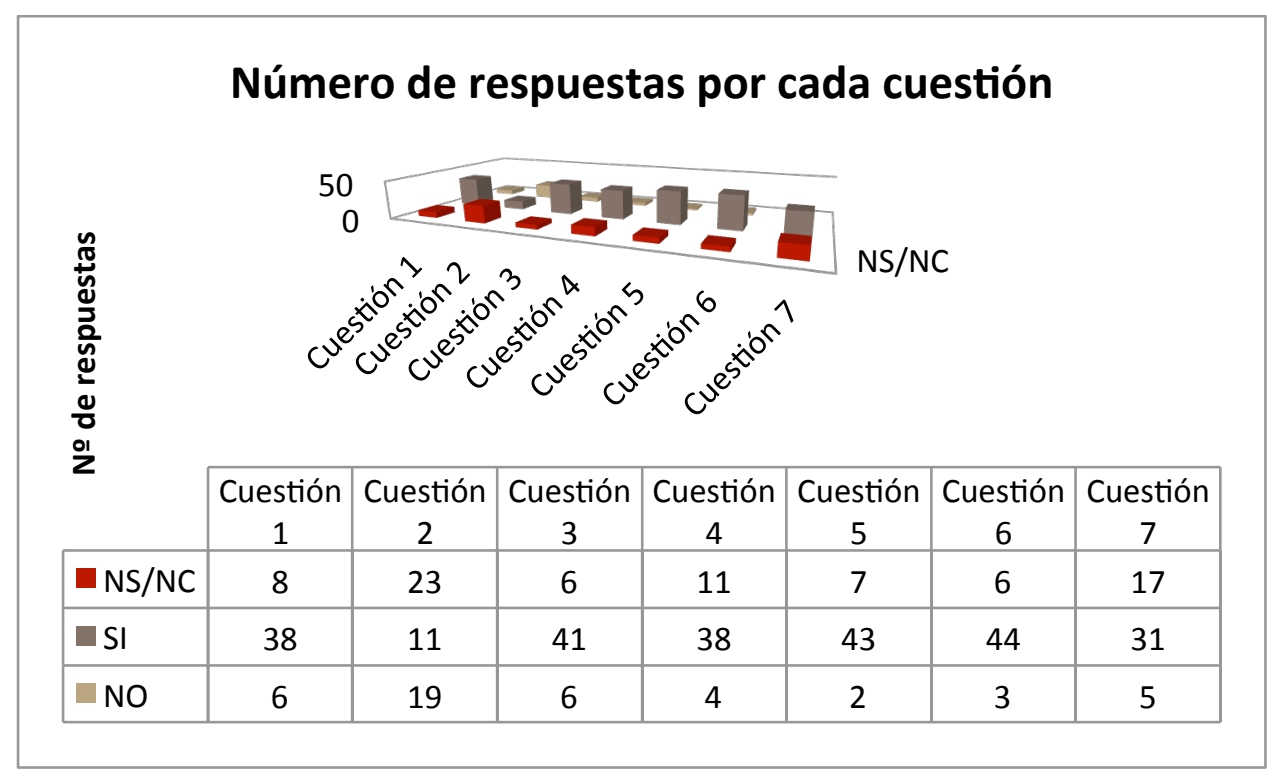

Figura 5. Gráfico de recuento de respuestas del cuestionario. 


\section{Discusión y conclusiones}

Con la implementación de este proyecto hemos conseguido una implicación y mejora de la competencia digital y el aprendizaje de nuevas herramientas TIC para estudiar el patrimonio histórico-artístico de un espacio territorial escogido previamente. Asimismo, y a partir de este itinerario didáctico diseñado con Google Earth, el alumnado del Grado de Educación Primaria delimitó en el entorno del río Guadalquivir a su paso por Córdoba, un recorrido con diferentes paradas que recogen los lugares que se van a visitar durante la salida. Con este planteamiento, la propuesta se ha programado para aunar innovación con investigación educativa a través de la práctica de campo y la de aula. De hecho, uno de los problemas a los que nos enfrentamos como docentes y en la formación de los futuros maestros es la falta de motivación de estos últimos por conocer la riqueza patrimonial de sus ciudades y saber cuál sería la herramienta didáctica más adecuada para transmitir esos conocimientos.

De todo lo dicho, se considera que al hablar de salidas y actividades que necesitan de un contacto directo con la naturaleza, la motivación es el aspecto más característico. Del mismo modo, al promover el contacto directo con los diferentes elementos históricos-artísticos y naturales, el alumnado en su formación como futuros docentes obtiene los conocimientos que debe trasladar en las aulas y fuera de éstas en su labor diaria. Otro de los aspectos que se promueve con esta experiencia es el conocimiento y difusión de la cultura andaluza, y con ello la puesta en valor de los recursos naturales y sociales que forman parte de nuestra identidad cultural. Por otro lado, la colaboración entre alumnos para sintetizar y compartir la información fue esencial con el objetivo de formalizar un aprendizaje significativo y asimismo favorecer el uso de las Nuevas Tecnologías en el ámbito educativo.

Por último, del análisis de los resultados pudimos observar que los estudiantes comprendieron las diferencias existentes entre recursos naturales y patrimoniales, además de la relación cultural que existe entre ambos. Las presentaciones realizadas por los estudiantes lograron describir a partir de lo investigado, una realidad que no es visible desde el punto de vista ambiental ni patrimonial, construyendo un discurso personal, implicado y construido a partir del trabajo colaborativo, que fue clave para poner en práctica las propuestas curriculares.

\section{Referencias}

Acosta, R., Martín, A.V. y Hernández, A. (2015). Propuesta de un modelo de Webquest para la enseñanza de geografía en Educación Secundaria con la aplicación Google Sites. EDUTEC, 52, 1-16.

Álvarez, A. (1990). Diseño cultural: Una aproximación ecológica a la educación desde el paradigma histórico-cultural. Infancia y Aprendizaje, 51-52, 41-77.

Antón, B. (2002). Educación Ambiental, conservar la naturaleza y mejorar el medio ambiente. Madrid: Ed. Escuela Española.

Ávila, R.M. (2003). La función del itinerario en la enseñanza y el aprendizaje del Patrimonio Histórico-Artístico. Íber: Didáctica de las ciencias sociales, geografía e historia, 36, 36-47.

Beltz, J. (2002). Materiales didácticos para la educación ambiental. Barcelona: Wolters Kluwer.
Calaf, R. (2003). Aprender arte en la ciudad: sensibilizar hacia el respeto y la valoración del patrimonio urbano. En Roser Calaf (Coord.) (2003). Arte para todos. Miradas para enseñar y aprender el patrimonio (103-136). Gijón: Trea.

Calaf, R. (2009). Didáctica del patrimonio: epistemología, metodología, y estudio de casos. Gijón: Trea.

Colom, A.J., Sarragona, J. y Vázquez, G. (1998). Educación ambiental y la conservación del patrimonio. Barcelona: Ariel.

Córcoles, J.E. (2010). Google Earth. Uso didáctico para la Escuela 2.0. Revista Digital: Sociedad de la información, 20, 9-19.

Cubero, R. (2000). Cómo trabajar con las ideas de los alumnos. Sevilla: Diada.

Cuenca, J.M. y Martín, M. (2009). La ciudad actual a través de la ciudad histórica. Cuadernos de Pedagogía, 394, 67-9. 
Cuenca, J.M. (2014). El papel del patrimonio en los centros educativos: hacia la socialización patrimonial. Tejuelo, 19, 7696.

Domingo, M. y Marqués, P. (2011). Aulas 2.0 y uso de las TIC en la práctica docente. Comunicar: Revista científica iberoamericana de comunicación y educación, 37, 169-175.

Domínguez, A. y López, R. (2014). Patrimonio, paisaje y educación: Formación inicial del profesorado y educación cívica del alumnado de primaria. Clio: History and History Teaching, 40, 1-26.

Estepa, J., Ávila, RM. y Ruiz, R. (2007). Concepciones sobre la enseñanza y difusión del patrimonio en las instituciones educativas y los centros de interpretación: estudio descriptivo. Enseñanza de las ciencias sociales: revista de investigación, 6, 75-94.

Fernández, V. (2005). Finalidades del patrimonio en educación. Investigación en la Escuela, 56, 7-18.

Fontal, O. (2006). Claves del patrimonio cultural del presente y desde el presente para abordar su enseñanza. Revista de Educación, 29, 9-31.

Friera, F. (2003). Itinerarios didácticos: teoría y experiencias en defensa del Patrimonio. En Cristina Fernández et al (Coords.) (2003). El Patrimonio y la Didáctica de las Ciencias Sociales (339-346). Cuenca: Universidad de Castilla-La ManchaAUPDCS.

González, N. (2008). Una investigación cualitativa y etnográfica sobre el valor educativo y el uso didáctico del patrimonio cultural. Enseñanza de las Ciencias Sociales, 7, 23-36.

Husillos, M. (2012). La Arqueología Virtual: Construyendo un puente entre la sociedad moderna y la escuela innovadora. Virtual Archaeology Review, 3, 36-39.

Insa, Y. (2004). Los itinerarios didácticos. Aula. Historia Social, 14, 87-91

Lata, S. y Castro, M. (2016). El Aprendizaje Cooperativo, un camino hacia la inclusión educativa. Revista Complutense de Educación, 27(3), 1085-1101.

Liceras, A. (2003). Observar e interpretar el paisaje. Estrategias didácticas. Granada: Grupo Editorial Universitario.

Martín, F. (1995). Bases teóricas de la Educación Ambiental: un modelo interdisciplinar. Revista Complutense de Educación, 6(2), 95-119.

Martín-Cáceres, M. y Cuenca, J.M. (2015). Educomunicación del patrimonio. Educatio Siglo XXI, 33(1), 33-54.

Novo, M. (2003). La Educación Ambiental. Bases éticas, conceptuales y metodológicas. Madrid: Universitas.

Prats, J. y Santacana, J. (2011). Por qué y para qué enseñar Historia. En Joaquim Prats y Joan Santacana (Coords.) (2011). Didáctica de la Geografía y la Historia (13-29). Barcelona: Grao.
Rivero, P. (2011). La arqueología virtual como fuente de materiales para el aula. Íber. Didáctica de las Ciencias Sociales, Geografía e Historia, 68, 17-24.

Rodríguez, M.E. y Bonilla, J. (2010). Inclusión y aplicación de las tecnologías en los centros educativos. Jaén: Formación Continuada Logos.

Rodríguez-Marín, F., Fernández-Arroyo, J. y García, JE. (2014). Las hipótesis de transición como herramienta didáctica para la Educación Ambiental. Enseñanza de las Ciencias, 32 (3), 303-318.

Travé, G. y Pozuelos, F.J. (1998). Investigar en el aula. Aportaciones para una didáctica innovadora. Huelva: Universidad de Huelva.

Vicent, N., Rivero, P., \& Feliu, M. (2015). Archeology and digital technologies in Heritage Education. Educatio siglo XXI, 33(1), 83-102.

Vilarrasa, A. (2003). Salir del aula. Reapropiarse del contexto. íber. Didáctica de las Ciencias Sociales, Geografía e Historia, $36,13-25$. 